\title{
Multiantenna Signal Processing for Cognitive Communications
}

\author{
(Invited Paper) \\ Symeon Chatzinotas, Shree Krishna Sharma, and Björn Ottersten \\ SnT - securityandtrust.lu, University of Luxembourg \\ Email:\{symeon.chatzinotas, shree.sharma, bjorn.ottersten\}@uni.lu
}

\begin{abstract}
Cognitive communications has attracted a large interest during the last decade due to spectrum scarcity. In combination with multiantenna techniques, cognitive communications have the ability to increase spectral efficiency by enabling the coexistence of a primary and secondary systems. In this paper, we focus in two specific cognitive approaches: a) Multiantenna Interference Alignment (IA) and b) Multiantenna Spectrum Sensing (SS). In the first case, we investigate how IA over multiple spatial dimensions can be exploited in order to lower harmful interference towards the primary system into acceptable levels. In the second case, we compare the sensing performance of different eigenvalue-based blind SS techniques. This paper concludes by presenting some interesting open problems in this area.

Index Terms-Multiple Antennas, Cognitive Communications,
\end{abstract} Spectrum Sensing, Interference Alignment

\section{INTRODUCTION}

Due to the booming market for wireless multimedia services, the demand for broadband wireless spectrum has been increased significantly. However, usable spectrum resources are becoming scarce due to spectrum segmentation policies and dedicated frequency allocations to various wireless systems. This scarcity has led to the concept of cognitive communications which allows for the coexistence of licensed and unlicensed systems over the same spectrum [1]. The cognitive techniques which are most commonly considered in the literature are Spectrum Sensing (SS), underlay, overlay and database techniques [2]. In SS only techniques, Secondary Users (SUs) are allowed to transmit whenever Primary Users (PUs) are inactive whereas in underlay techniques, SUs are allowed to transmit provided that they respect an interference constraint which guarantees the Quality of Services (QoS) of PUs.

In existing literature, multiple antennas have been considered in a wide range of applications such as beamforming, spatial diversity/multiplexing, Interference Alignment (IA) etc. In the context of spectral coexistence of two systems, multiple antennas can be used for several purposes such as SS [3]-[5], cognitive beamforming [6], [7] and cognitive IA [8], [9]. In this paper, we focus on two important applications of multiple antennas to exploit the underutilized primary spectrum: (i) IA and (ii) SS.

The coexistence of two systems within the same spectrum can be modeled as a Cognitive Radio (CR) network with interference channels between primary and secondary systems. When the strength of secondary interference to the primary is comparable to the desired signal, treating as noise is not an option because of the interference constraints involved while decoding. The IA technique was firstly proposed in [10] and channel capacity as well as degrees of freedom (dof) for the interference channel have been analysed. The IA technique has been shown to achieve the dofs for a range of interference channels [11], [12]. Its principle is based on aligning the interference on a signal subspace with respect to the non-intended receivers so that it can be easily filtered out by sacrificing some signal dimensions. The exact number of needed dimensions and the precoding vectors to achieve IA are rather cumbersome to compute, but a number of approaches have been presented in the literature towards this end [13]-[15]. In the context of cognitive communications, the IA in an underlay mode has received important attention recently in the cognitive radio research community [16] [17]. The fundamental assumptions in this technique are that there are multiple available dimensions (space, frequency, time or code) and that the Secondary Transmitter (ST) is aware of the Channel State Information (CSI) towards the Primary Receivers (PR). In this paper, we apply this technique in the spectral coexistence scenario of outdoor femto cells and a macro cell uplinks to mitigate the interference of femto cell User Terminals (UTs) towards the macrocell Base Station (BS). For this purpose, we consider static, uncoordinated and coordinated IA techniques. The proposed IA techniques are compared to no-mitigation technique as well as to a resource sharing approach in terms of primary rate protection ratio.

Spectrum Sensing is an important process used to acquire the spectrum awareness required by the CRs. Several SS techniques such as Energy Detection (ED), matched filter and cyclostationary feature detection techniques have been proposed in the literature [18]-[20]. The later two techniques require the prior knowledge of the PU's signal to make the decision about the presence or absence of the PU signal [2]. Although ED does not require any prior knowledge of PU's signal, the performance of this technique is susceptible to noise covariance uncertainty [21]. Furthermore, different multi-antenna techniques have been considered in the literature to enhance the SS efficiency in wireless fading channels [5], [22]-[24]. In most of these methods, the properties of the eigenvalues of the received signal's covariance matrix have been considered using recent results from advances in Random Matrix Theory (RMT) [25], [26]. Eigenvalue based SS does 
not require any prior information of the PU's signal and it outperforms ED techniques, especially in the presence of noise covariance uncertainty [22]. In this context, we compare the performance of different eigenvalue-based blind SS techniques using an asymptotic approach for multi-antenna sensing.

The remainder of this paper is structured as follows: Section II presents the system and signal models and the proposed multiantenna IA technique with some numerical results. Section III presents the multi-antenna SS techniques with the focus on multi-antenna eigenvalue-based sensing and some numerical results. Section IV concludes this paper by presenting research challenges in this area.

\section{Multiantenna Interference Alignment}

In this application scenario, we consider the problem of mitigating interference from femtocell terminals towards the macrocell BS while operating both macro and femto uplink systems within the same spectrum.

\section{A. System and Signal Model}

We consider a single macrocell BS receiving signals from a set of PUs and a number of femto cells $(N)$ which operate over the same coverage area receiving signals from a set of the SUs as shown in Fig. 1. The femto cells can cooperate through a backhaul link and jointly decode the received signals [27]-[31]. After scheduling, we consider that for a single slot one macro UT and $N$ femto cell UTs transmit simultaneously over a common spectrum. The interference from femtocell UTs towards the macrocell BS needs to be suppressed to protect the reception of PU signals from harmful interference. We consider that the macro UT has $M$ antennas while the BS, femto cell UTs and the femto cell Access Points (APs) have $L=M+1$ antennas. We further assume that femto cell UTs have CSI towards the macro BS. This CSI can be easily measured with the help of the macrocell pilot signals. In addition, there is a predefined vector $\mathbf{v}$ which is known by both femtocell UTs and the macro BS. We employ precoding at the femto cell UTs so that the received secondary signals at the macro BS are all aligned across vector $\mathbf{v}$. In the considered scenario, the femtocell UTs have to be aware of the CSI and vector $\mathbf{v}$ to perform the precoding and the macro BS needs only to perform spatial filtering over vector $\mathbf{v}$ without having additional awareness.

The received signal at the macro BS can be written as:

$$
\mathbf{y}_{1}=\mathbf{H x}+\sum_{i=1}^{N} \mathbf{F}_{i} \mathbf{x}_{i}+\mathbf{z}_{1},
$$

where $\mathbf{y}_{1}$ is the $L \times 1$ received symbol vector, $\mathbf{x}$ is the $M \times 1$ transmitted symbol vector from the Primary Transmitter (PT), $\mathbf{x}_{i}$ is the $L \times 1$ transmitted symbol vector from the $i$-th ST and $\mathbf{z}_{1}$ is the receiver noise. All inputs $\mathbf{x}, \mathbf{x}_{i}$ are assumed to be Gaussian and obey the following sum-power constraints: $\mathbb{E}\left[\mathbf{x}^{\dagger} \mathbf{x}\right] \leq \gamma_{p s} M$ and $\mathbb{E}\left[\mathbf{x}_{i}^{\dagger} \mathbf{x}_{i}\right] \leq \gamma_{s s} L, \gamma_{p s}$ being the transmit $\mathrm{SNR}^{a}$ of the PT and $\gamma_{s s}$ being the transmit SNR of the ST. The $L \times M$ matrix $\mathbf{H}$ represents the channel gains between the PR and the PT while the $L \times L$ matrix $\mathbf{F}_{i}$ represents the channel

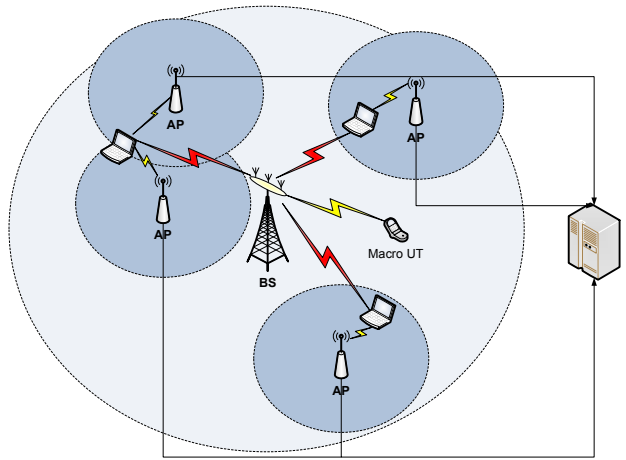

Fig. 1. System model for the considered coexistence scenario.

gains between the PR and $i$-th ST . To simplify notations, we group all $\mathbf{F}_{i}$ into a single $L \times N L$ matrix $\mathbf{F}=\left[\mathbf{F}_{1} \ldots \mathbf{F}_{N}\right]$. The received signal at the joint processor of the SRs is:

$$
\mathbf{y}_{2}=\sum_{i=1}^{N} \tilde{\mathbf{F}}_{i} \mathbf{x}_{i}+\tilde{\mathbf{H}} \mathbf{x}+\mathbf{z}_{2},
$$

where $\mathbf{y}_{2}$ is the $N L \times 1$ received symbol vector and $\mathbf{z}_{2}$ is the receiver noise. The $N L \times M$ channel matrix $\tilde{\mathbf{H}}$ represents the channel gains between all SRs and the PT while the $N L \times L$ channel matrix $\tilde{\mathbf{F}}_{i}$ represents the channel gains between all SRs and the $i$-th ST. To simplify notations, we group all $\tilde{\mathbf{F}}_{i}$ into a single $N L \times N L$ matrix $\tilde{\mathbf{F}}=\left[\tilde{\mathbf{F}}_{1} \ldots \tilde{\mathbf{F}}_{N}\right]$.

We consider a Multiple Input Multiple Output (MIMO) Rayleigh channel whose power is scaled according to a power-law path loss model i.e., $\mathbf{H}=\alpha \mathbf{G}$, where $\alpha$ is the path loss coefficient between the BS and the macro UT and $\mathbf{G}$ is a $L \times M$ random matrix with complex circularly symmetric (c.c.s.) independent identically distributed (i.i.d.) elements representing Rayleigh fading coefficients. Similarly, $\mathbf{F}_{i}=\alpha_{i} \mathbf{J}_{i}$, where $\alpha_{i}$ is the path loss coefficient between BS and $i$-th femto-cell $\mathrm{UT}$ and $\mathbf{J}_{i}$ is a $L \times L$ random matrix with i.i.d. c.c.s. elements representing channel coefficients between femto-cell BS and the $i$-th femto-cell UT. As a result, $\mathbf{F}=\left(\boldsymbol{\alpha}^{T} \otimes \mathbb{I}_{L \times L}\right) \odot \mathbf{J}$ with $\boldsymbol{\alpha}=\left[\alpha_{1} \ldots \alpha_{N}\right]^{T}$ and $\mathbf{J}$ is a $L \times N L$ random matrix with i.i.d. c.c.s. elements. In addition, $\tilde{\mathbf{H}}=\left(\boldsymbol{\beta} \otimes \mathbb{I}_{L \times M}\right) \odot \tilde{\mathbf{G}}$, where $\boldsymbol{\beta}=\left[\beta_{1} \ldots \beta_{N}\right]^{T}$ includes path loss coefficients between all APs and macro UT and $\tilde{\mathbf{G}}$ denotes a $N L \times M$ random matrix with i.i.d. c.c.s. elements. Similarly, $\tilde{\mathbf{F}}_{i}=\left(\boldsymbol{\beta}_{i} \otimes \mathbb{I}_{L \times L}\right) \odot \tilde{\mathbf{J}}_{i}$, where $\boldsymbol{\beta}_{i}$ contains the path loss coefficient between all APs and the $i$-th femto-cell UT and $\tilde{\mathbf{J}}_{i}$ represents a $N L \times L$ random matrix with i.i.d. c.c.s. elements. As a result, $\tilde{\mathbf{F}}=\left(\mathbf{B} \otimes \mathbb{I}_{L \times L}\right) \odot \tilde{\mathbf{J}}$ with $\mathbf{B}=\left[\boldsymbol{\beta}_{1} \ldots \boldsymbol{\beta}_{N}\right]$ and $\tilde{\mathbf{J}}$ is a $N L \times N L$ random matrix with i.i.d. c.c.s. elements.

\section{B. IA Techniques}

The IA is employed at all the STs towards the PR and interference is filtered out at the PR by using the IA vector $\mathbf{v}$. Let us assume a $L \times 1$ non-zero reference vector $\mathbf{v}$ along which the interference should be aligned. It should be noted that STs are assumed to know the alignment direction $\mathbf{v}$ and to have perfect own CSI about the channel coefficients $\mathbf{F}_{i}$ towards 
the PR. In this context, the following precoding scheme is employed to align the interference:

$$
\mathbf{x}_{i}=\mathbf{w}_{i} x_{i}=\left(\mathbf{F}_{i}\right)^{-1} \mathbf{v} v_{i} x_{i} .
$$

where $\|\mathbf{v}\|^{2}=L$ and $\mathbb{E}\left[\mathbf{x}_{i}^{\dagger} \mathbf{x}_{i}\right] \leq L \gamma$. the scaling variable $v_{i}$ is needed to ensure that the input power constraint is not violated for each ST. This precoding results in unit multiplexing gain and is by no means the optimal IA scheme, but it serves as a tractable way of evaluating the IA performance. Following this approach, the cochannel interference can be expressed as:

$$
\sum_{i=1}^{N} \mathbf{F}_{i} \mathbf{x}_{i}=\sum_{i=1}^{N} \mathbf{F}_{i}\left(\mathbf{F}_{i}\right)^{-1} \mathbf{v} v_{i} x_{i}=\mathbf{v} \sum_{i=1}^{N} v_{i} x_{i} .
$$

It can be easily seen that interference has been aligned across the reference vector and it can be removed using a $M \times L$ zero-forcing filter $\mathbf{Q}$ designed so that $\mathbf{Q}$ is a truncated unitary matrix [11] and $\mathbf{Q v}=\mathbf{0}$. After filtering, the $M \times 1$ received signal vector at the PR can be expressed as: $\overline{\mathbf{y}}_{1}=\overline{\mathbf{H}} \mathbf{x}+\overline{\mathbf{z}}_{1}$, where $\overline{\mathbf{H}}=\mathbf{Q H}$ is the $M \times M$ filtered channel matrix. The received signal at the joint processor of the SRs is:

$$
\overline{\mathbf{y}}_{2}=\sum_{i=1}^{N} \overline{\mathbf{F}}_{i} x_{i}+\tilde{\mathbf{H}} \mathbf{x}+\mathbf{z}_{2},
$$

where $\overline{\mathbf{F}}_{i}=\tilde{\mathbf{F}}_{i}\left(\mathbf{F}_{i}\right)^{-1} \mathbf{v} v_{i}$ are the equivalent $N L \times 1$ channel matrices including precoding. To simplify notations we group all $\overline{\mathbf{F}}_{i}$ into a single $N L \times N$ matrix $\overline{\mathbf{F}}=\left[\overline{\mathbf{F}}_{1} \ldots \overline{\mathbf{F}}_{N}\right]$. In the following paragraphs, we describe three different IA approaches ${ }^{1}$.

1) Static Approach: The alignment direction is predefined and does not depend on the channel state. This is a simple solution assuming no coordination in the network. The disadvantage is that the IA direction may be aligned with one of the strong eigenvectors of the random PR-PT channel and a large amount of received power may be filtered out.

2) Coordinated Approach: The selection of the alignment direction takes place at the PR and is subsequently communicated to STs. It is assumed that the channel coherence time is adequate for the alignment direction to be fed back and used by STs.

3) Uncoordinated Approach: This approach assumes no coordination between two systems. Furthermore, the STs are aware of their CSI towards the PR but have no information about the CSI of the PT. STs select an alignment direction which maximizes the secondary throughput and the PR is responsible for sensing the alignment direction and applying the appropriate filter.

\section{Numerical Results}

The primary only throughput in the absence of secondary can be written as:

$$
\mathrm{C}_{p o}=\mathbb{E}\left[\log \operatorname{det}\left(\mathbf{I}_{L}+\frac{\gamma_{p s}}{M} \mathbf{H H}^{\dagger}\right)\right],
$$

where $\mathbf{I}_{L}$ is the identity matrix of dimension $L$ and $\gamma_{p s}$ represents the SNR at the transmit antenna of the primary

\footnotetext{
${ }^{1}$ The mathematical details on these techniques can be found in [8].
}

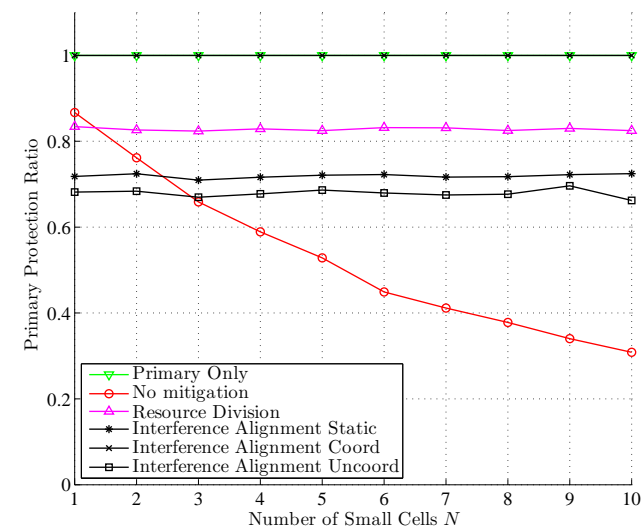

Fig. 2. Primary protection ratio vs. number of femto cells $N$.

system. The primary throughput in presence of secondary can be written as:

$$
\mathrm{C}_{p s}=\mathbb{E}\left[\log \operatorname{det}\left(\mathbf{I}_{M}+\frac{\gamma_{p s}}{M} \overline{\mathbf{H}} \overline{\mathbf{H}}^{\dagger}\right)\right],
$$

where $\overline{\mathbf{H}}$ is the equivalent channel matrix after IA filtering. The primary rate protection ratio can be denoted by $\mathrm{PR}$ and is defined as: $\mathrm{PR}=\frac{\mathrm{C}_{p s}}{\mathrm{C}_{p o}}$. Figure 2 presents the $\mathrm{PR}$ versus $N$ for a set of typical parameters described in [8]. It should be especially noted that the coordinated IA approach fully protects the primary rate as expected while other IA approaches preserve roughly $70 \%$ and the resource division preserves $82 \%$ of the primary rate. Furthermore, all the techniques except nomitigation preserve a constant protection rate with increasing $N$, while the performance of no-mitigation technique degrades monotonically. In addition, IA techniques achieve roughly the same system spectral efficiency (SE) as Resource Division, where SE is defined as the sum of primary and secondary SEs.

\section{Multiantenna Spectrum Sensing}

We consider a multi-antenna assisted CR node with $M$ number of antennas as in [3]. Let $N$ be the number of observations collected by each CR node in the time duration of $\tau$. We consider a generic signal model assuming the presence of multiple PUs, while the signal model for the case of single PU can be considered as a specific case. We assume that channel remains constant during the period of sensing and the transmitted PU symbols are i.i.d. complex circularly symmetric (c.c.s.) Gaussian symbols. A single observation of the $M \times 1$ received signal $\mathbf{y}$ in presence of $K$ PUs can be written as:

$$
\mathbf{y}=\sum_{i=1}^{K} \mathbf{h}_{i} s_{i}+\mathbf{z}=\mathbf{H s}+\mathbf{z}
$$

where $\mathbf{s}$ is $K \times 1$ transmitted signal i.e. $\mathbf{s}=\left[s_{1}, s_{2}, \ldots, s_{K}\right]^{T}$, with $s_{i}$ being a Gaussian symbol with power $p_{i}=\mathbb{E}\left[s_{i}^{2}\right]$. The $M \times K$ channel matrix $\mathbf{H}$ includes the channel coefficients between PUs and $M$ receive nodes i.e. $\mathbf{H}=\left[\begin{array}{llll}\mathbf{h}_{1} & \mathbf{h}_{2} & \ldots & \mathbf{h}_{K}\end{array}\right]$, $\mathbf{z}$ is $M \times 1$ Gaussian noise vector with zero mean and variance $\sigma^{2}$. After collecting $N$ samples for each receiving 
node, the $M \times N$ received signal matrix $\mathbf{Y}$ can be written as: $\mathbf{Y}=\left[\mathbf{y}_{1}, \mathbf{y}_{2} \ldots \mathbf{y}_{N}\right]$. Let us denote the hypotheses of the presence and absence of the PU signal by $\mathbb{H}_{1}$ and $\mathbb{H}_{0}$ respectively. The binary hypothesis testing problem for deciding the presence of a PU signal can be written as:

$$
\mathbb{H}_{0}: \mathbf{Y}=\mathbf{Z}, \quad \mathbb{H}_{1}: \mathbf{Y}=\mathbf{H S}+\mathbf{Z}
$$

where $\mathbf{S}$ is the $K \times N$ transmitted signal and $\mathbf{Z}$ is the $M \times N$ Gaussian noise. Let us define sample covariance matrices of received signal and noise as: $\mathbf{R}_{\mathbf{Y}}(N)=\frac{1}{N} \mathbf{Y} \mathbf{Y}^{H}$ and $\mathbf{R}_{\mathbf{Z}}(N)=\frac{1}{N} \mathbf{Z} \mathbf{Z}^{H}$. Under noise only hypothesis, $\mathbf{R}_{\mathbf{Y}}(N)=$ $\mathbf{R}_{\mathbf{Z}}(N)$. It can be noted that since $\mathbf{Z} \sim \mathcal{C N}(0, \mathbf{I}), \mathbf{Z Z}^{H}$ follows an uncorrelated Wishart distribution [22] i.e. $\mathbf{Z Z}^{H} \sim$ $\mathcal{W}_{M}(\boldsymbol{\Sigma}, N)$, where $\boldsymbol{\Sigma}=\frac{\mathbb{E}\left[\mathbf{Z Z}^{H}\right]}{N}$.

\section{A. Blind SS Techniques}

1) Scaled Largest Eigenvalue Technique: The decision for SLE method can be made on the basis of following binary hypothesis testing:

$$
\text { decision }= \begin{cases}\mathbb{H}_{0}, & \text { if } \frac{\lambda_{\max }\left(\mathbf{R}_{\mathbf{Y}}(N)\right)}{\frac{1}{M} \operatorname{tr}\left\{\mathbf{R}_{\mathbf{Y}}(N)\right\}} \leq T_{S L E}^{\infty} \\ \mathbb{H}_{1}, & \text { otherwise }\end{cases}
$$

where $T_{S L E}^{\infty}$ is the asymptotic threshold for the SLE method, which can be written as [5]: $T_{S L E}^{\infty}=(1+1 / \sqrt{\beta})^{2}$, where $\beta=N / M$.

2) SCN based Technique: The decision for SCN based technique can be made in the following way:

$$
\text { decision }= \begin{cases}\mathbb{H}_{0}, & \text { if } \frac{\lambda_{\max }\left(\mathbf{R}_{\mathbf{Y}}(\mathrm{N})\right)}{\lambda_{\min }\left(\mathbf{R}_{\mathbf{Y}}(\mathrm{N})\right)} \leq T_{S C N}^{\infty} \\ \mathbb{H}_{1}, & \text { otherwise }\end{cases}
$$

where $T_{S C N}^{\infty}$ is the asymptotic threshold for the SCN method which is given by [32], [33]: $T_{S C N}^{\infty}=\frac{(1+\sqrt{\beta})^{2}}{(1-\sqrt{\beta})^{2}}$.

3) Spherical Test Method: The test statistic for this method is calculated as the ratio of the geometric and arithmetic mean of all eigenvalues:

$$
T_{S T}=\frac{\left(\operatorname{det}\left(\mathbf{R}_{\mathbf{Y}}(N)\right)\right)^{1 / M}}{\frac{1}{M} \operatorname{tr}\left(\mathbf{R}_{\mathbf{Y}}(N)\right)}=\frac{\left(\prod_{i=1}^{M} \lambda_{i}\right)^{1 / M}}{\frac{1}{M} \Sigma_{i=1}^{M} \lambda_{i}}
$$

The binary hypothesis testing based on this method can be expressed as:

$$
\text { decision }= \begin{cases}\mathbb{H}_{0}, & \text { if } T_{S T} \geq T_{S T}^{\infty} \\ \mathbb{H}_{1}, & \text { otherwise }\end{cases}
$$

where $T_{S T}^{\infty}$ represents the asymptotic threshold for the ST method, which is given by [5]: $T_{S T}^{\infty}=\exp (-1-(\beta-1) \log (1-1 / \beta))$

4) John's Detection Method: The test statistic for this method is given as the ratio of the quadratic mean over the arithmetic mean of all eigenvalues i.e. $T_{J}=\frac{\sqrt{\sum_{i=1}^{M} \lambda_{i}^{2}}}{\sum_{i=1}^{M} \lambda_{i}}$. The binary hypothesis testing based on this method can be expressed as:

$$
\text { decision }= \begin{cases}\mathbb{H}_{0}, & \text { if } T_{J} \leq T_{J}^{\infty} \\ \mathbb{H}_{1}, & \text { otherwise }\end{cases}
$$

where $T_{J}^{\infty}$ represents the asymptotic threshold for JD detector which is given by [5]: $T_{J}^{\infty}=\sqrt{1+1 / \beta}$.

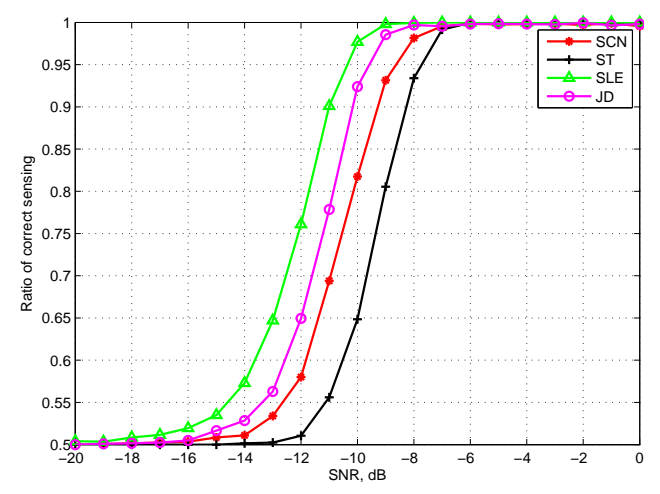

Fig. 3. Ratio of correct sensing versus SNR for different techniques in Rayleigh fading channel $(\beta=10, N=100)$

\section{B. Numerical Results}

Figure 3 shows the ratio of correct sensing versus SNR for different techniques with parameters $\beta=10, N=100$ in Rayleigh fading channel assuming the channel remains constant across during the period of sensing. From the result, it can be noted that SLE detector performs the best among other detectors and JD detector performs slightly worse than the SLE detector and better than SCN-based and ST detectors. During simulation, it was observed that the same performance ordering was preserved for multiple user scenario and for Rician fading channel conditions.

\section{CONCLUSIONS AND Discussion}

This paper has presented two important applications of multiple antennas in the context of cognitive communication. The IA technique has been considered in order to mitigate the interference from secondary femto UTs to the macro BS equipped with MIMO transceivers. The primary protection ratio of the IA techniques have been compared with non-IA techniques and it is noted that the coordinated IA technique perfectly protects the primary rate, while achieving the SE of Resource Division. Furthermore, the comparison of different blind eigenvalue based SS techniques have been presented by using an asymptotic analysis under noise only hypothesis. It has been observed that the performance of the SLE detector is optimal for a range of scenarios, followed by JD, SCN and ST.

In the coordinated IA technique, it is assumed that the ST-PR channel as well as the CSI of the PT is perfectly known to the ST. Although different techniques exist in acquiring the channel information, there always arises the uncertainty of perfect CSI. In this context, exploring the IA techniques which can provide better primary protection in the absence of channel knowledge in one important research challenge. Furthermore, applying IA in the downlink is more challenging since interference would have to be aligned across all receivers. In the context of SS, there remain many open research challenges such as distributed multiantenna sensing, collaborative multiantenna sensing, applying the asymptotic analysis to finite cases with higher accuracy etc. Furthermore, investigating the effect of noise and channel correlation in the performance of different SS techniques is an open issue. 


\section{REFERENCES}

[1] S. K. Sharma, S. Chatzinotas, and B. Ottersten, "Satellite cognitive communications: Interference modeling and techniques selection," in 6th ASMS/SPSC Conf., Sept. 2012.

[2] A. Goldsmith, S. Jafar, I. Maric, and S. Srinivasa, "Breaking spectrum gridlock with cognitive radios: An information theoretic perspective," Proc. of the IEEE, vol. 97, no. 5, pp. 894 -914, May 2009.

[3] P. Wang, J. Fang, N. Han, and H. Li, "Multiantenna-assisted spectrum sensing for cognitive radio," IEEE Trans. Veh. Technol., vol. 59, no. 4, pp. $1791-1800$, May 2010 .

[4] S. K. Sharma, S. Chatzinotas, and B. Ottersten, "Eigenvalue based sensing and SNR estimation for cognitive radio in presence of noise correlation," IEEE Trans. Veh. Technol., vol. 62, no. 8, pp. 1-14, 2013.

[5] S. Chatzinotas, S. K. Sharma, and B. Ottersten, "Asymptotic analysis of eigenvalue-based blind spectrum sensing techniques," in IEEE ICASSP, May 2013.

[6] S. K. Sharma, S. Chatzinotas, and B. Ottersten, "Transmit beamforming for spectral coexistence of satellite and terrestrial networks," in Int. Conf. CROWNCOM 2013, July 2013.

[7] S. K. Sharma, S. Chatzinotas, and B. Ottersten, "Spatial filtering for underlay cognitive SatComs," in 5th Int. Conf. Personal Satellite Services (PSATS), June 2013.

[8] S. Chatzinotas and B. Ottersten, "Cognitive interference alignment between small cells and a macrocell," in 19th Int. Conf. Telecommun., april 2012, pp. $1-6$.

[9] S. K. Sharma, S. Chatzinotas, and B. Ottersten, "Interference alignment for spectral coexistence of heterogeneous networks," EURASIP Journal On Wireless Communications and Networking, vol. 46, no. , pp. 1-14, 2013.

[10] V. Cadambe and S. Jafar, "Interference alignment and spatial degrees of freedom for the $\mathrm{k}$ user interference channel," in IEEE Int. Conf. on Commun., may 2008, pp. 971 -975.

[11] - "Interference alignment and the degrees of freedom of wireless X networks," IEEE Trans. Inf. Th., vol. 55, no. 9, pp. 3893 -3908, sept. 2009.

[12] S. Jafar and S. Shamai, "Degrees of freedom region of the MIMO X channel," IEEE Trans. Inf. Th., vol. 54, no. 1, pp. 151 -170, jan. 2008.

[13] K. Gomadam, V. Cadambe, and S. Jafar, "Approaching the capacity of wireless networks through distributed interference alignment," in IEEE GLOBECOM, nov. 2008, pp. $1-6$.

[14] C. M. Yetis, T. Gou, S. A. Jafar, and A. H. Kayran, "On feasibility of interference alignment in MIMO interference networks," IEEE Trans. on Signal process., vol. 58, no. 9, pp. 4771-4782, 2010.

[15] R. Tresch, M. Guillaud, and E. Riegler, "On the achievability of interference alignment in the K-user constant MIMO interference channel," in IEEE/SP 15th Workshop Statistical Signal Processing, aug. 2009, pp. $277-280$.

[16] H. Zhou, T. Ratnarajah, and Y.-C. Liang, "On secondary network interference alignment in cognitive radio," in IEEE Symp. New Frontiers in Dynamic Spectrum Access Networks, may 2011, pp. 637 -641.

[17] S. Kaimaletu, R. Krishnan, S. Kalyani, N. Akhtar, and B. Ramamurthi, "Cognitive interference management in heterogeneous femto-macro cell networks," in IEEE Int. Conf. on Commun., june 2011, pp. 1 -6.

[18] T. Yucek and H. Arslan, "A survey of spectrum sensing algorithms for cognitive radio applications," IEEE Commun. Surveys Tutorials, vol. 11, no. 1 , pp. $116-130$, quarter 2009.

[19] S. K. Sharma, S. Chatzinotas, and B. Ottersten, "Exploiting polarization for spectrum sensing in cognitive satcoms," in 7th Int. Conf. CROWNCOM, June 2012.

[20] S. K. Sharma, S. Chatzinotas, and B. Ottersten, "Spectrum sensing in dual polarized fading channels for cognitive satcoms," in IEEE Globecom Conf., Dec. 2012.

[21] R. Tandra and A. Sahai, "SNR walls for signal detection," IEEE J. of Selected Topics in Signal Process., vol. 2, no. 1, pp. 4 -17, Feb. 2008.

[22] Y. Zeng and Y. chang Liang, "Eigenvalue-based spectrum sensing algorithms for cognitive radio," IEEE Trans. Commun., vol. 57, no. 6, pp. $1784-1793$, June 2009.

[23] W. Zhang, G. Abreu, M. Inamori, and Y. Sanada, "Spectrum sensing algorithms via finite random matrices," IEEE Trans. Commun., vol. 60, no. 1 , pp. $164-175$, Jan. 2012.

[24] A. Kortun, T. Ratnarajah, M. Sellathurai, C. Zhong, and C. Papadias, "On the performance of eigenvalue-based cooperative spectrum sensing for cognitive radio," IEEE J. Selected Topics in Signal Process., vol. 5, no. 1, pp. $49-55$, Feb. 2011.

[25] A. M. Tulino and S. Verdu, "Random matrix theory and wireless communications," Foundations and Trends in Communications and Information Theory, vol. 1, no. 1, pp. 1-182, 2004.

[26] R. Couillet and M. Debbah, Random Matrix Methods for Wireless Communications, 1st ed. Cambridge University Press, 2011.

[27] S. Chatzinotas, M. A. Imran, and C. Tzaras, "Optimal information theoretic capacity of the planar cellular uplink channel," in IEEE 9th Workshop on Signal Processing Advances in Wireless Communications (SPAWC'08), Pernambuco, Brazil, Jul 2008, pp. 196-200.

[28] S. Chatzinotas, M. Imran, and R. Hoshyar, "On the multicell processing capacity of the cellular MIMO uplink channel in correlated Rayleigh fading environment," IEEE Trans. Wireless Commun., vol. 8, no. 7, pp. 3704-3715, July 2009.

[29] S. Chatzinotas, M. Imran, and C. Tzaras, "On the capacity of variable density cellular systems under multicell decoding," IEEE Commun. Letter, vol. 12, no. 7, pp. 496 - 498, Jul 2008.

[30] — "The effect of user distribution on a cellular multiple-access channel," in Third International Conference on Communications and Networking in China (ChinaCom'08), Hangzhou, China, August 2008, pp. 95-99.

[31] S. Chatzinotas and B. Ottersten, "Free probability based capacity calculation of multiantenna Gaussian fading channels with cochannel interference," Physical Communication, vol. 4, no. 3, pp. 206 - 217, 2011, recent Advances in Cooperative Communications for Wireless Systems. [Online]. Available: http://www.sciencedirect.com/science/article/pii/S1874490711000188

[32] L. Cardoso, M. Debbah, P. Bianchi, and J. Najim, "Cooperative spectrum sensing using random matrix theory," in 3rd Int. Symp. Wireless Pervasive Computing, May 2008, pp. $334-338$.

[33] S. K. Sharma, S. Chatzinotas, and B. Ottersten, "The effect of noise correlation on fractional sampling based spectrum sensing," in Int. Conf. Commun. (ICC), June 2013, pp. $1182-1187$. 